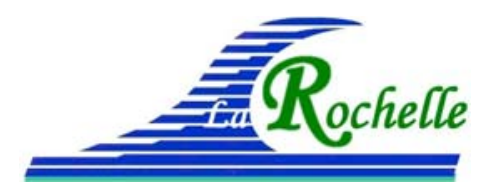

XVèmes Journées Nationales Génie Côtier - Génie Civil

La Rochelle, 29 au 31 mai 2018

DOI:10.5150/jngcgc.2018.066

(C) Editions Paralia CFL

disponible en ligne - $h$ ttp://www.paralia.fr - available online

\title{
Caractérisation statistique des durées d'arrêt et de fonctionnement des fermes éoliennes offshores
}

\author{
Olivier ORCEL ${ }^{1}$, Philippe SERGENT ${ }^{1}$
}

\author{
1. Cerema Eau Mer et Fleuves, 134 rue de Beauvais, 60280 Margny lès Compiègne CS \\ 60321, France. \\ Olivier.Orcel@cerema.fr ; Philippe.Sergent@cerema.fr
}

\begin{abstract}
Résumé : Afin d'analyser l'intégration de la production énergétique des fermes éoliennes offshores dans le réseau électrique européen, nous avons déterminé les lois de probabilité des durées de fonctionnement et d'arrêt des éoliennes. Afin de faciliter l'exploitation de ces données, nous avons obtenu des distributions théoriques de type Gamma qui s'ajustent aux lois réelles. Nous avons pu ainsi obtenir les périodes de retour annuelles et décennales pour ce qui concerne les durées d'arrêt et de fonctionnement. Nous avons également calculé les moyennes, les écarts-types sur chaque site étudié, selon les années, les mois et les saisons, puis leur évolution selon la vitesse minimale d'utilisation.
\end{abstract}

Mots clés : Eolienne offshore, Données de vent, Distribution Gamma.

\section{Introduction}

La lutte contre le réchauffement climatique s'inscrit comme un enjeu de société. Pour la promouvoir, il convient de favoriser les énergies renouvelables et en particulier l'éolien offshore. Il est cependant nécessaire d'analyser le caractère intermittent de cette énergie renouvelable en estimant les durées de fonctionnement et d'arrêt.

A partir d'échantillons de données de vitesses de vent obtenues au pas horaire pendant 31 ans sur 9 sites offshores issues du CFSR (Climate Forecast System Reanalysis), nous déterminons les lois réelles des durées de fonctionnement continu (vitesses comprises entre $5 \mathrm{~m} / \mathrm{s}$ et $25 \mathrm{~m} / \mathrm{s}$ ) et d'arrêt continu des éoliennes. Contrairement à l'usage courant (BAILE, 2010 ; SBAI et al., 1994 ; MORGAN et al., 2011 ; FAIDA, 2010), qui préconise les lois de Weibull et parfois gaussiennes ou elliptiques pour modéliser la distribution de la vitesse du vent, nous privilégions les distributions Gamma pour modéliser ici la distribution de la durée de fonctionnement continu et d'arrêt continu des éoliennes. Bien que les lois d'Erlang soient les lois les plus utilisées pour les problèmes de files d'attente avec des variables qui modélisent des distributions de durées inter évènements, nous avons retenu dans notre travail les lois Gamma car elles généralisent ces lois d'Erlang et s'ajustent mieux aux données réelles des éoliennes.

Les lois Gamma retenues présentent l'intérêt d'une plus grande facilité de manipulation que les données brutes et permettent de calculer les périodes de retour. L'obtention des 


\section{Thème 5 - Énergies et ressources marines}

périodes de retour d'arrêt annuelles, décennales à partir des lois Gamma permettront en particulier de dimensionner des sources d'énergie complémentaires. Nous calculons également les moyennes de durée de fonctionnement ou d'arrêt des éoliennes avec leur écart-type selon les sites, les années, les mois, les saisons ou la vitesse minimale d'utilisation.

\section{Présentation de la méthode}

\subsection{Présentation des sites}

Les neuf sites étudiés 2729, 4772, 2970, 3283, 3919, 5027, 7762, 8408 et 9868 sont situés sur la carte ci-dessous.

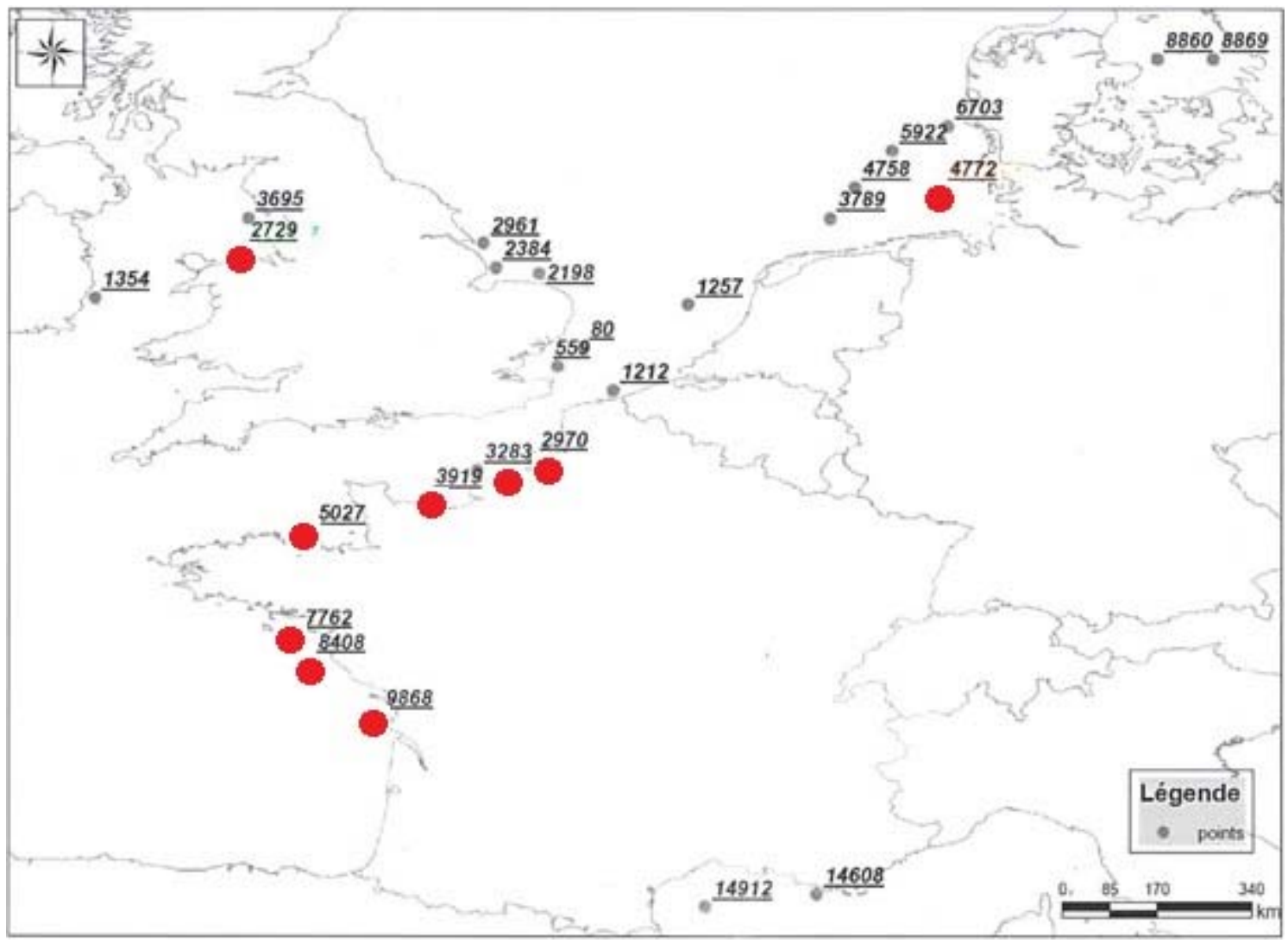

Figure 1. Carte des sites étudiés.

\subsection{Description de la méthode}

Nous disposons de données de vitesses au pas horaire sur 31 ans pour 9 sites. Ce sont des données issues du CFSR (Climate Forecats System Reanalysis). Ces données ont été comparées aux données du modèle ERA-Interim et à des données in-situ (THIEBAUD et al., 2018). Les deux modèles sont globalement cohérents malgré des écarts importants en mer Baltique pour la vitesse de vent à 10 mètres de hauteur issue des deux modèles. Les données CFSR ont également été comparées à des mesures de vent 


\section{XVèmes Journées Nationales Génie Côtier - Génie Civil \\ La Rochelle, 29 au 31 mai 2018}

en mer du Nord et en mer Baltique. L'écart moyen est de l'ordre de 1,3 m/s pour les deux points de mesure.

Considérant que les éoliennes offshores fonctionnent pour des vitesses comprises entre $5 \mathrm{~m} / \mathrm{s}$ et $25 \mathrm{~m} / \mathrm{s}$, nous sélectionnons les instants compris dans l'intervalle [ $5 \mathrm{~m} / \mathrm{s}, 25$ $\mathrm{m} / \mathrm{s}$ ]. Nous pouvons ainsi déterminer les différentes durées d'utilisation et d'arrêt, ainsi que les durées d'inter-arrivées. A partir de ces échantillons de données, nous obtenons une loi réelle des durées de fonctionnement ou d'arrêt des éoliennes. Nous représentons ces dernières ainsi que les lois théoriques Gamma.

\subsection{Modélisation de la distribution des fréquences d'arrivée de vent}

Les notations sont les suivantes :

$\mathbf{D}_{\mathbf{i}}$ : Début du ième fonctionnement de l'éolienne : v $\in[5 \mathrm{~m} / \mathrm{s}, 25 \mathrm{~m} / \mathrm{s}]$.

$F_{\mathbf{i}}$ : Fin du $\mathrm{i}^{\text {ème }}$ fonctionnement de l'éolienne : v $\in[0,5 \mathrm{~m} / \mathrm{s}[\mathrm{U}] 25 \mathrm{~m} / \mathrm{s},+\infty[$.

$\mathbf{U}_{\mathrm{i}}=\mathbf{F}_{\mathrm{i}}-\mathbf{D}_{\mathrm{i}}$ : Durée du i ème fonctionnement (ou utilisation) de l'éolienne (v $\in[5 \mathrm{~m} / \mathrm{s}, 25$ $\mathrm{m} / \mathrm{s}])$.

$\mathbf{C}_{\mathbf{i}}=\mathbf{D}_{\mathbf{i}}-\mathbf{F}_{\mathrm{i}-1}$ : Durée du ième arrêt des éoliennes (v $\in[0,5 \mathrm{~m} / \mathrm{s}[\mathrm{U}] 25 \mathrm{~m} / \mathrm{s},+\infty[)$.

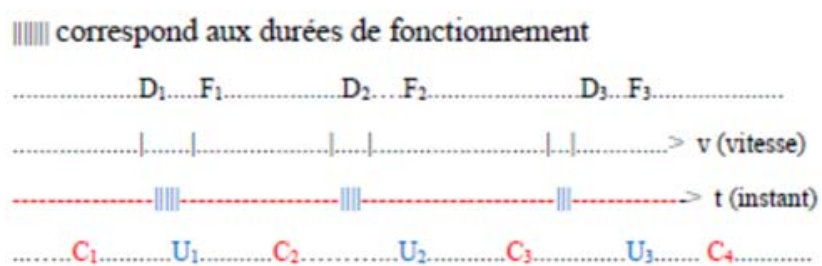

Figure2. Schématisation de la modélisation du vent.

\subsection{Ajustement par la loi Gamma}

La distribution de la durée de fonctionnement ou d'arrêt des éoliennes peut être décrite par la loi Gamma. Une distribution Gamma (MANOUKIAN, 1986 ; TASSI, 2004) est caractérisée par deux paramètres qui affectent respectivement la forme et l'échelle de sa représentation graphique. Une variable aléatoire $X$ suit une loi Gamma de paramètre $k$ et $\theta$ (strictement positifs), ce que l'on note aussi $\mathrm{X} \sim \Gamma(k, \theta)$ si sa fonction de densité de probabilité peut se mettre sous la forme :

$$
f(x ; k, \theta)=\frac{x^{k-1} e^{-\frac{x}{\theta}}}{\Gamma(k) \theta^{k}}, k>0, \theta>0
$$

avec $\Gamma$ la fonction Gamma d'Euler. La loi Gamma a pour espérance $E=k \theta$. La loi Gamma a pour variance : $\mathrm{V}=k \theta^{2}$. Les paramètres de la loi théorique s'obtiennent par les relations : $\theta=V / E(1 / \theta$ paramètre d'intensité) et $k=E / \theta$ (paramètre de forme).

\section{Résultats et discussion}




\section{Thème 5 - Énergies et ressources marines}

Après avoir décrit les données et proposé la distribution de Gamma, nous présentons cidessous quelques résultats.

\subsection{Espérances et écarts-types sur les différents sites}

Pour des vitesses d'utilisation comprises dans l'intervalle $[5 \mathrm{~m} / \mathrm{s}, 25 \mathrm{~m} / \mathrm{s}]$, nous déterminons les espérances $E$ et écarts-types $\sigma$ des durées de fonctionnement et d'arrêt en heures pour les différents sites.

Tableau 1. Espérances et écarts-types en heure des durées d'utilisation et d'arrêt des éoliennes en fonction des sites (sur 31 ans).

\begin{tabular}{llllllllll}
\hline Sites & $\mathbf{2 7 2 9}$ & $\mathbf{4 7 7 2}$ & $\mathbf{2 9 7 0}$ & $\mathbf{3 2 8 3}$ & $\mathbf{3 9 1 9}$ & $\mathbf{5 0 2 7}$ & $\mathbf{7 7 6 2}$ & $\mathbf{8 4 0 8}$ & $\mathbf{9 8 6 8}$ \\
\hline $\boldsymbol{E}(\boldsymbol{U})$ & 24 & 43 & 27 & 32 & 24 & 26 & 20 & 23 & 17 \\
$\boldsymbol{E}(\boldsymbol{C})$ & 18 & 11 & 14 & 13 & 14 & 13 & 14 & 11 & 13 \\
$\boldsymbol{\sigma}(\boldsymbol{U})$ & 35 & 63 & 43 & 49 & 40 & 43 & 32 & 35 & 26 \\
$\boldsymbol{\sigma}(\boldsymbol{C})$ & 24 & 11 & 17 & 16 & 16 & 13 & 15 & 11 & 13 \\
\hline
\end{tabular}

Nous constatons sur le tableau 1 que la moyenne de la durée de fonctionnement est de l'ordre d'une journée pour une moyenne d'arrêt d'une demi-journée. L'écart-type du de la durée de fonctionnement est de l'ordre de deux jours et d'une demi-journée pour la durée d'arrêt. Le site 4772, qui est localisé en Mer du Nord, présente des caractéristiques de fonctionnement plus intéressantes que les autres sites pour le fonctionnement mais les durées d'arrêt sont en revanche proches des autres sites.

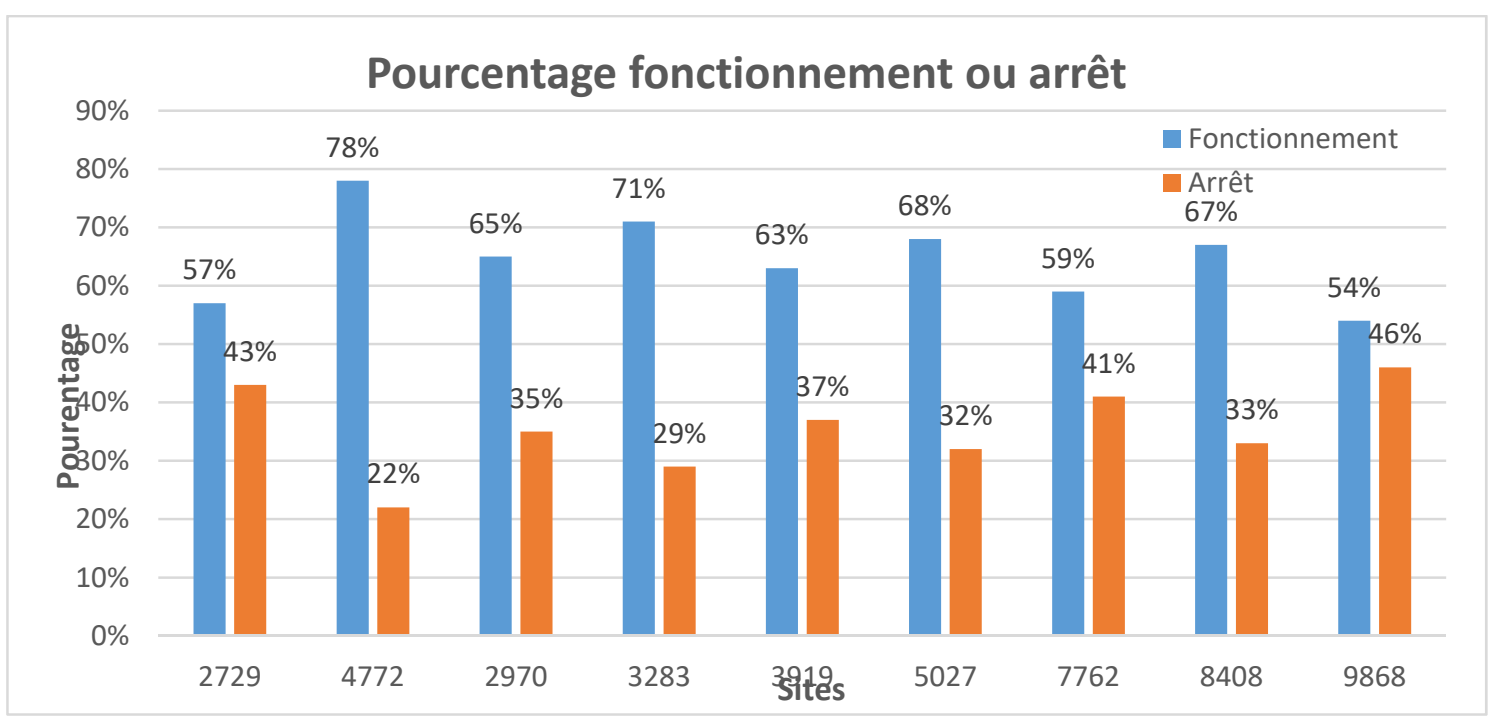

Figure 2. Pourcentages de fonctionnement et d'arrêt des sites avec une vitesse minimale de $5 \mathrm{~m} / \mathrm{s}$. 


\section{XVèmes Journées Nationales Génie Côtier - Génie Civil \\ La Rochelle, 29 au 31 mai 2018}

Les résultats en pourcentage de la figure 2 indiquent que les sites fonctionnent en moyenne 63\% du temps (avec un maximum de 78\%) en Mer du Nord et un minimum de $54 \%$ devant l'île d'Oléron sur la côte Atlantique. Nous nous intéressons plus particulièrement aux pourcentages d'arrêt qui passent de $22 \%$ à $46 \%$ et qui peuvent donc doubler par rapport à la Mer du Nord.

\subsection{Evolution des moyennes et écarts-types selon la vitesse minimale}

Nous avons fait évoluer la vitesse minimale pour l'échantillon 2729. Nous constatons que plus la vitesse minimale décroît plus la durée moyenne et l'écart-type de durée de fonctionnement croissent. Pour une vitesse minimale de $5 \mathrm{~m} / \mathrm{s}$, nous avons une moyenne d'un jour, qui progresse à 2 jours à $3 \mathrm{~m} / \mathrm{s}$ puis à 3 jours à $2 \mathrm{~m} / \mathrm{s}$. Inversement, les durées d'arrêt moyennes passent d'une demi-journée à $3 \mathrm{~m} / \mathrm{s}$ à 1 jour à $6 \mathrm{~m} / \mathrm{s}$. Afin de pouvoir représenter les courbes des lois théoriques relatives aux différentes vitesses minimales, nous avons déterminé les paramètres des lois gamma.

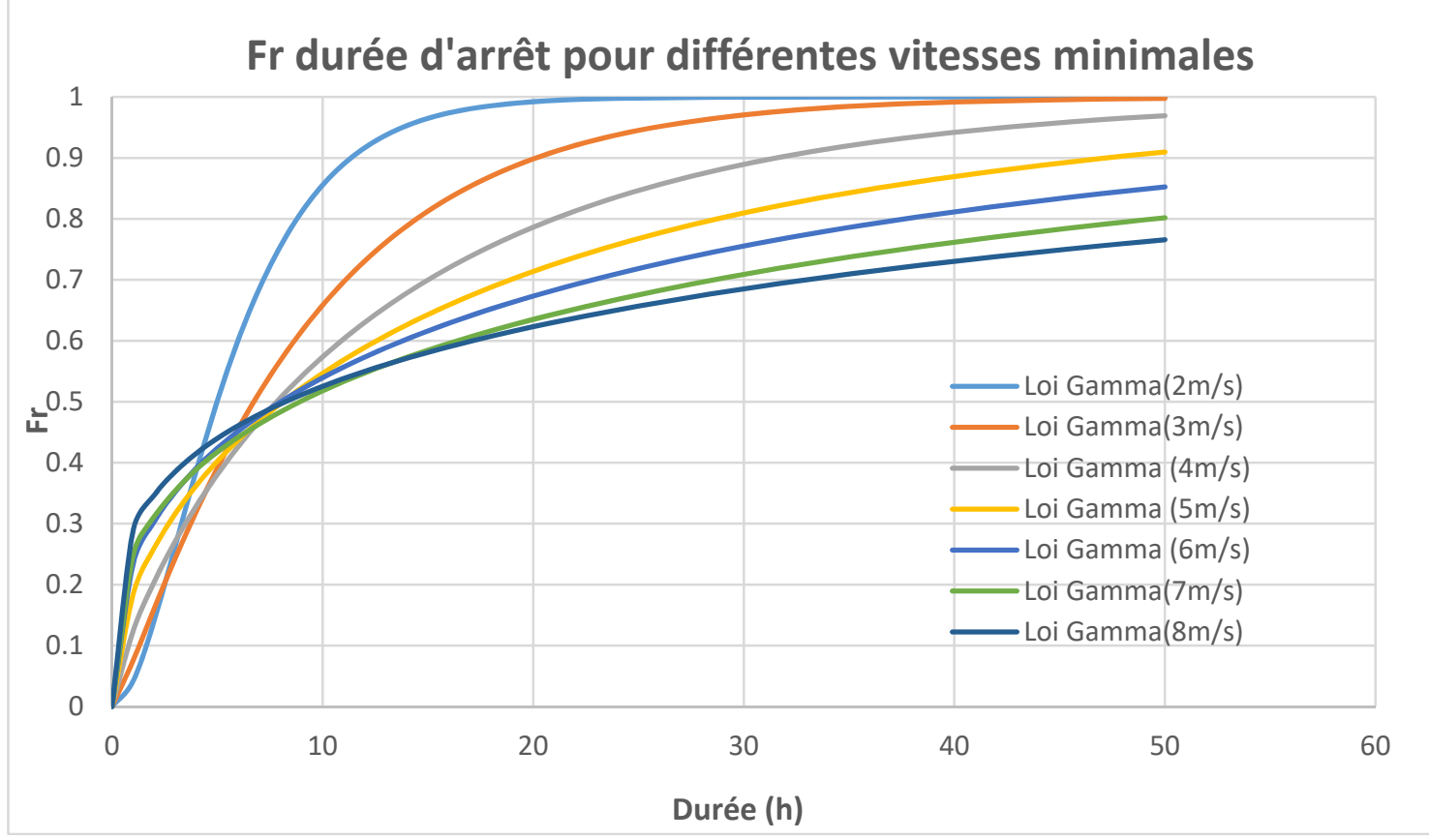

Figure 3. Fonction de répartition des durées d'utilisation des éoliennes en fonction de la vitesse minimale.

Comme les espérances et les écarts-types, les fonctions de répartition évoluent fortement en fonction des vitesses minimales sur la figure 3. Nous remarquons que, lorsque la vitesse minimale augmente, le pourcentage de petites durées d'arrêt augmente ainsi que les grandes durées. La vitesse minimale de fonctionnement conditionne donc fortement les arrêts.

\subsection{Analyse inter-annuelle}




\section{Thème 5 - Énergies et ressources marines}

Nous avons choisi une vitesse de $4 \mathrm{~m} / \mathrm{s}$ pour l'analyse des variations inter-annuelles. Pour une vitesse de vent dans l'intervalle $[4 \mathrm{~m} / \mathrm{s}, 25 \mathrm{~m} / \mathrm{s}]$, nous déterminons ainsi les espérances et écarts-types d'utilisation et d'arrêt des éoliennes du site 2729 en mer d'Irlande. Les moyennes de durée de fonctionnement et d'arrêt varient selon les années jusqu'à $30 \%$.

\subsection{Moyennes et écarts-types mensuels}

Pour l'année 1979, nous déterminons les moyennes et écarts-types pour les différents mois et pour l'échantillon 2729.

Tableau 2. Tableau des espérances et des écarts-types en heure des durées d'utilisation et d'arrêt des éoliennes par mois pour l'année 1979 (pour le site $n^{\circ} 2729$ ).

\begin{tabular}{lllllllllllll}
\hline Mois & $\mathbf{0 1}$ & $\mathbf{0 2}$ & $\mathbf{0 3}$ & $\mathbf{0 4}$ & $\mathbf{0 5}$ & $\mathbf{0 6}$ & $\mathbf{0 7}$ & $\mathbf{0 8}$ & $\mathbf{0 9}$ & $\mathbf{1 0}$ & $\mathbf{1 1}$ & $\mathbf{1 2}$ \\
\hline $\boldsymbol{E}(\boldsymbol{U})$ & 29 & 33 & 41 & 15 & 11 & 11 & 24 & 14 & 25 & 26 & 44 & 27 \\
$\boldsymbol{E}(\boldsymbol{C})$ & 11 & 14 & 8 & 14 & 18 & 40 & 36 & 18 & 14 & 20 & 12 & 11 \\
$\boldsymbol{\sigma}(\boldsymbol{U})$ & 32 & 52 & 73 & 25 & 12 & 15 & 36 & 17 & 24 & 31 & 31 & 25 \\
$\boldsymbol{\sigma}(\boldsymbol{C})$ & 11 & 1 & 8 & 15 & 15 & 51 & 37 & 15 & 10 & 22 & 12 & 11 \\
\hline
\end{tabular}

Les mois froids (de septembre à mars) ont une moyenne de durée de fonctionnement continu de l'ordre d'un à deux jours alors que les mois chauds (d'avril à août) ont une moyenne de durée de fonctionnement continu d'une demi-journée environ. Inversement, les mois froids (de novembre à mai) ont une moyenne durée d'arrêt continu d'une demijournée alors que les mois chauds (de juin à octobre) ont une moyenne de durée d'arrêt continu de un à deux jours.

\section{Obtention de distributions théoriques et des périodes de retour}

\subsection{Lois théoriques des différents sites et validation}

Nous présentons sur le tableau 3 les différentes lois gamma avec leurs caractéristiques pour les durées de fonctionnement et d'arrêt des éoliennes. Les paramètres $k$ et $\theta$ ont été obtenus à partir de l'espérance et de la variance empiriques données par le tableau 1 et les relations : $k=E / \theta$ et $\theta=\sigma^{2} / E$.

Tableau 3. Tableau des lois Gamma $G(k, \theta)$ ( $U$ : fonctionnement, $C$ : arrêt)

\begin{tabular}{lllllllllll}
\hline Sites & $\mathbf{2 7 2 9}$ & $\mathbf{4 7 7 2}$ & $\mathbf{2 9 7 0}$ & $\mathbf{3 2 8 3}$ & $\mathbf{3 9 1 9}$ & $\mathbf{5 0 2 7}$ & $\mathbf{7 7 6 2}$ & $\mathbf{8 4 0 8}$ & $\mathbf{9 8 6 8}$ \\
\hline $\boldsymbol{U}$ & $G(0.47,51)$ & $G(0.42,97)$ & $G(0.4,68)$ & $G(0.43,75)$ & $G(0.36,67)$ & $G(0.39,51)$ & $G(0.43,51)$ & $G(0.43,53)$ & $G(0.43,40)$ \\
$\boldsymbol{C}$ & $G(0.56,32)$ & $G(1,11)$ & $G(0.67,21)$ & $G(0.66,20)$ & $G(0.87,18)$ & $G(0.87,16)$ & $G(0.87,16)$ & $G(1,11)$ & $G(1,13)$ \\
\hline
\end{tabular}




\section{XVèmes Journées Nationales Génie Côtier - Génie Civil \\ La Rochelle, 29 au 31 mai 2018}

La validation des lois théoriques des différents sites a pu se faire avec le test du chi2. Nous représentons sur la figure 4 les courbes théorique et réelle avec des données brutes de la loi de de durée de fonctionnement (ou d'utilisation) des éoliennes.

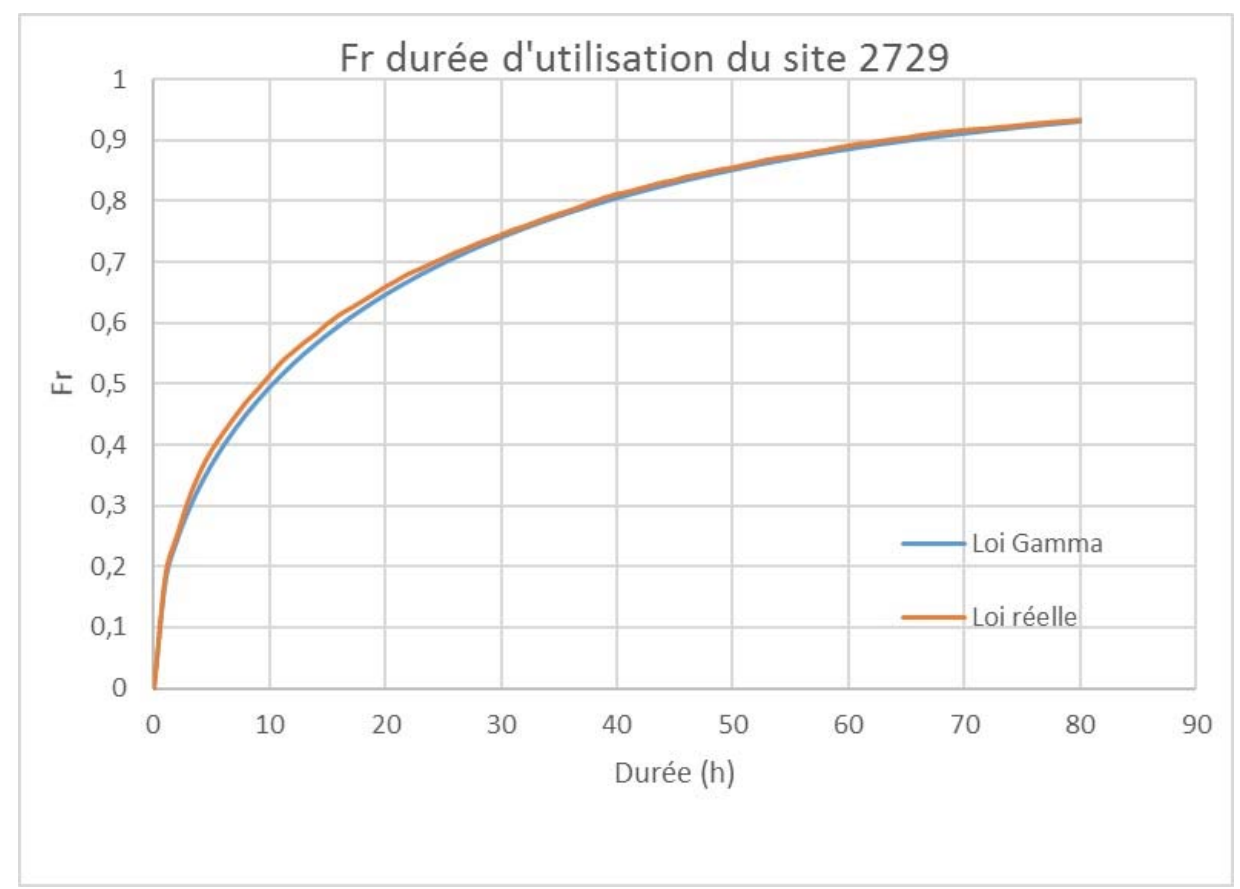

Figure 4. Loi réelle et théorique de durée de fonctionnement du site 2729 avec une vitesse minimale de $5 \mathrm{~m} / \mathrm{s}$.

\subsection{Périodes de retour annuelles ou décennales pour les sites}

Il s'agit d'obtenir la durée $x$ de fonctionnement continu en heures (ou d'arrêt continu) tel que $G(x ; k, \theta)=1-1 / n$ avec $G$ la fonction de répartition de la loi Gamma du site et $n$ le nombre moyen de périodes de fonctionnement continu (ou d'arrêt continu) sur un an ou dix ans (10 $\mathrm{x} n$ annuel). Les paramètres $k$ et $\theta$ dépendent du site et aussi de l'analyse (fonctionnement ou arrêt). Le tableau 4 présente $n$ annuel ainsi que les valeurs de $G$ proches de 1 qui nous intéressent pour l'estimation de ces valeurs extrêmes de fonctionnement continu (ou d'arrêt continu) $x$ en heures.

Tableau 4. Tableau des fréquences annuelles ou décennales pour les 9 sites.

\begin{tabular}{llllllllll}
\hline Sites & $\mathbf{2 7 2 9}$ & $\mathbf{4 7 7 2}$ & $\mathbf{2 9 7 0}$ & $\mathbf{3 2 8 3}$ & $\mathbf{3 9 1 9}$ & $\mathbf{5 0 2 7}$ & $\mathbf{7 7 6 2}$ & $\mathbf{8 4 0 8}$ & $\mathbf{9 8 6 8}$ \\
\hline $\boldsymbol{n}$ annuel & 203 & 164 & 218 & 201 & 232 & 233 & 264 & 264 & 301 \\
$\boldsymbol{G}(\boldsymbol{x})$ annuel & 0.995 & 0.994 & 0.995 & 0.995 & 0.996 & 0.996 & 0.996 & 0.996 & 0.996 \\
$\boldsymbol{G}(\boldsymbol{x})$ décennal & 0.9995 & 0.9994 & 0.9995 & 0.9995 & 0.9996 & 0.9996 & 0.9996 & 0.9996 & 0.9997 \\
\hline
\end{tabular}




\section{Thème 5 - Énergies et ressources marines}

L'inversion de la fonction de répartition $G$ du site avec le langage $\mathrm{R}$ nous donne les durées $x$ en heures de fonctionnement continu (ou d'arrêt continu) maximales annuelles et décennales qui sont représentées sur la figure 5 .

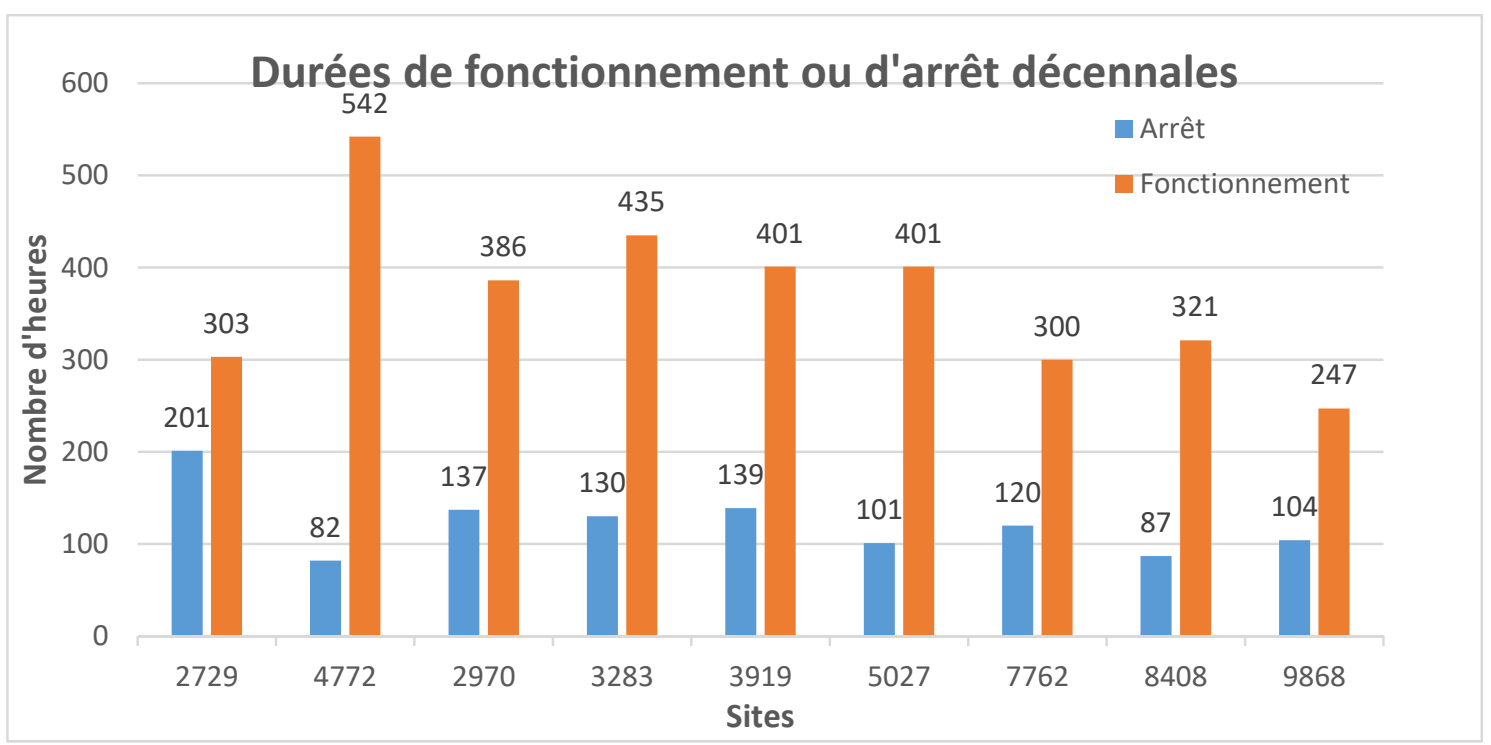

Figure 5. Durées de fonctionnement ou d'arrêt décennales pour les 9 sites (en heures).

La durée de fonctionnement maximale décennale varie de 10 à 22,5 jours avec une moyenne de 15,5 jours. Plus intéressant, la durée d'arrêt maximale décennale varie de 3,5 jours à 8,5 jours avec un minimum en mer du Nord et un maximum en mer d'Irlande. En France, la durée d'arrêt décennale est d'environ 5 jours. La durée d'arrêt maximale annuelle varie de 2 jours à 5 jours avec un minimum en mer du Nord et un maximum en mer d'Irlande. En France, la durée d'arrêt maximale annuelle est d'environ 3 jours. Ces résultats sont à nuancer dans un sens ou un autre, car nous prenons des vitesses de fonctionnement comprises entre $5 \mathrm{~m} / \mathrm{s}$ et $25 \mathrm{~m} / \mathrm{s}$ à $10 \mathrm{~m}$ de hauteur. En effet, en abaissant le seuil de la vitesse minimale, nous avons vu que le fonctionnement est plus important. Mais même si l'éolienne fonctionne, la production à ces vitesses reste faible. L'ordre de grandeur trouvé peut donc être utile pour caractériser les situations critiques d'intermittence ou dimensionner un système de stockage.

\section{Conclusion et perspectives}

Nous avons déterminé la moyenne et l'écart type des durées d'arrêt et de fonctionnement pour les 9 sites, selon les années, les mois et la vitesse minimale de fonctionnement. Ce travail a permis l'obtention de distributions théoriques de type Gamma, qui s'ajustent aux lois réelles de durée de fonctionnement ou d'arrêt pour des 


\section{XVèmes Journées Nationales Génie Côtier - Génie Civil La Rochelle, 29 au 31 mai 2018}

éoliennes offshores et qui ont permis l'obtention des périodes de retour d'arrêt annuelles et décennales.

Ce travail pourra être étendu aux situations avec des durées de fonctionnement courtes entre plusieurs longues durées d'arrêt.

\section{Références bibliographiques}

BAILE R. (2010). Analyse et modélisation multifractales de vitesses de vent. Application à la prévision de la ressource éolienne. Thèse de l'université de Corse. URL : https://tel.archives-ouvertes.fr/tel-00604139

FAIDA H. (2010). Etude et analyse des données du vent en vue du dimensionnement au système d'énergie éolienne. Cas d'un site au nord du Maroc. Revue des Energies Renouvelables, Vol. 13(3), pp 477-483.

MANOUKIAN E.B. (1986). Guide de statistique appliquée. Herman, éditeurs des sciences et des arts, $202 \mathrm{p}$.

MORGAN E.C., LACKNER M., VOGEL R.M., BAISE L.G. (2011). Probability distributions for offshore wind speeds. Revue Energy Conversion and Management, Vol. 52, pp 15-26. https://doi.org/10.1016/j.enconman.2010.06.015

SBAI A., MOUHDI A., ADOUK N., PAUL F. (1994). Modélisation de la vitesse du vent et calcul du potentiel éolien du Maroc occidental. Revue La Météorologie, $8^{\text {ème }}$ série, $\mathrm{n}^{\circ} 5$, pp 54-60. https://doi.org/10.4267/2042/53402

TASSI P. (2004). Méthodes statistiques. Economica, collection économie et statistiques avancées, $482 \mathrm{p}$.

THIEBAUD L., TIBERI-WADIER A.L., BEAUDOUIN Y. (2018). Analyse de données de vent en Europe pour caractériser le potentiel éolien en mer. XV ${ }^{\text {èmes }}$ Journées Génie Côtier Génie Civil, La Rochelle. 
Thème 5 - Énergies et ressources marines 